\title{
Single nucleotide polymorphisms of complement component 5 and periodontitis
}

\author{
L. Chai ${ }^{1}$, Y.-Q. Song ${ }^{2}$, K.-Y. Zee ${ }^{1,3}$ and W. K. Leung ${ }^{1}$ \\ ${ }^{1}$ Faculty of Dentistry, The University of Hong Kong, ${ }^{2}$ Department of Biochemistry, Li Ka \\ Shing Faculty of Medicine, The University of Hong Kong, Hong Kong SAR, China, ${ }^{3}$ Current \\ address: Periodontics, Westmead Centre for Oral Health, Westmead Hospital, New South \\ Wales, Australia
}

Key words: Complement C5; Periodontitis; Polymorphism, Genetic; Smoking

Running title: SNPs of complement C5 and periodontitis

Date of resubmission: May 11, 2009

\section{Corresponding author:}

W. Keung Leung

Faculty of Dentistry, The University of Hong Kong, Room 3B39, Prince Philip Dental Hospital, 34 Hospital Road, Hong Kong SAR, China.

Tel: +852 28590417;

Fax: +852 28587874;

e-mail: ewkleung@hkucc.hku.hk 
Chai L, Song Y-Q, Zee K-Y and Leung WK. Single nucleotide polymorphisms of complement component 5 and periodontitis. J Periodontal Res

Background and Objective: Polymorphisms of host defence genes might increase one's risks for periodontitis. This study investigated whether tagging single-nucleotide polymorphisms (SNPs) of the gene encoding complement component 5 (C5) are associated with periodontitis in a Hong Kong Chinese population.

Material and Methods: Eleven tagging SNPs of 229 patients with at least moderate periodontitis and 207 control subjects without periodontitis were genotyped with an i-plexGOLD MassARRAY mass-spectrometry system (Sequenom, San Diego, CA, USA).

Results: Genotype AG of SNP rs17611 was more prevalent in the periodontitis patient group than in the controls $(54.6 \%$ vs. $41.7 \%, p=0.007)$. The haplotype CGCA of the haplotype block consisting of rs1035029, rs17611, rs25681, and rs992670 was significantly associated with periodontitis in a dominant model $(p=0.001)$. The SNP rs17611 showed high linkage disequilibrium with rs1035029, rs25681, and rs992670. Smoking was also significantly associated with periodontitis $(p=0.006)$.

Conclusion: The tagging SNP rs17611 of the C5 gene and smoking may be associated with periodontitis among the Hong Kong Chinese population. 
Periodontitis is a complex human inflammatory disease that is caused by dental plaque, and its clinical manifestations are determined by both environmental influences on and genetic makeup of affected individuals. Polymorphisms of host defence genes might also increase the risk of periodontitis (1).

The complement system is a lytic effector system that protects the host against microbial pathogens and acts as a key link between innate and specific immune responses $(2,3)$, and has been implicated in the pathogenesis of periodontitis. The complement components have been detected in the gingival cervical fluid collected from the periodontitis patients $(4,5)$. It has been reported that the increased complement cleavage was associated with increased severity of inflammation and periodontal destruction (6). The complement component deficiencies seemed associated with severe chronic periodontitis (7). Complement component 5 (C5) is a pivotal element in the complement system therefore could take part in the pathogenesis of periodontitis. It is cleaved by $\mathrm{C} 5$ convertase to yield the $\mathrm{C} 5 \mathrm{~b}$ fragment and the anaphylatoxin $\mathrm{C} 5 \mathrm{a}$. The latter binds to the $\mathrm{G}$ protein-coupled receptor $\mathrm{C} 5 \mathrm{aR}$ to trigger intracellular signalling, which results in chemotaxis, respiratory burst, and release of proinflammatory mediators from granulocytes $(8,9)$. The $\mathrm{C} 5 \mathrm{~b}$ fragment combines with complement components $\mathrm{C} 6$ and $\mathrm{C} 7$ to initiate the formation of the membrane attack complex (MAC) in the membrane of invading microorganisms (10). 
Because haplotype and tagging polymorphisms of C5 may be associated with some chronic inflammatory diseases such as liver fibrosis, rheumatoid arthritis, and bronchial asthma (11-16), C5 polymorphisms may also be important in chronic inflammatory oral diseases such as periodontitis. Yet, published data on the relevance of C5 polymorphisms in periodontitis are not yet available.

The objective of this study was to screen tagging single-nucleotide polymorphisms (SNPs) of the C5 gene in Hong Kong Chinese patients with moderate to severe periodontitis and in periodontitis-free controls. We investigated whether there were any genetic variations in the C5 genes of these two groups and tested whether such variations, together with other possible risk factors such as smoking status, age and sex, were associated with periodontitis.

\section{Materials and methods}

This case-control study was approved by the Ethics Committee of the Faculty of Dentistry, The University of Hong Kong. Written informed consent was obtained from all participants. 


\section{Study participants}

Participants were recruited from new patients attending the Primary Care Clinic, Prince Philip Dental Hospital (PPDH), Faculty of Dentistry, The University of Hong Kong, from May 2005 to August 2007. Patients' records and radiographs were screened within 1 month of first attendance and potentially eligible patients were invited to attend a clinical examination. Demographic information and medical and dental histories were obtained from patients' records, supplemented by information obtained during the day of the clinical examination. Race and ethnicity were self-reported, with a participant being considered Chinese if his or her biological parents, grandparents, and great grandparents were all reported to be ethnic Chinese. Smoking history was self-reported; patients who currently smoked or who had quit within 12 months were considered to be smokers and those who had never smoked or who had quit for more than 12 months were considered to be non-smokers. All participants needed to be systemically healthy, so those with systemic conditions including cardiovascular diseases, hypertension, liver diseases, kidney diseases, blood disorders, diabetes mellitus, autoimmune diseases, and malignant tumours were excluded, as were pregnant females.

Periodontitis-free and periodontitis groups were defined on the basis of both radiographs and findings of clinical examinations. Cases or periodontitis subjects 
were Chinese patients aged 18 to 60 years with radiographic evidence of at least moderate periodontitis, according to the following criteria: the orthopantomogram (OPG) taken at the first visit to PPDH before recruitment showed more than $50 \%$ alveolar bone loss at more than $30 \%$ of sites, as measured with a Schei ruler without using the $1 \mathrm{~mm}$ space (each tooth contributed to a mesial and a distal site) (17), and subsequent clinical periodontal examination showed at least two teeth in each quadrant that had a probing depth of $\geq 5 \mathrm{~mm}$ and that bled on probing. All the periodontitis subjects showed clinical and radiographic signs of attachment loss and bone loss, respectively, and were further classified into aggressive periodontitis or chronic periodontitis as described follows. According to the 1999 periodontal disease classification criteria (18), patients $\leq 35$ years of age and systemic healthy but experiencing over $30 \%$ sites with over $5 \mathrm{~mm}$ clinical attachment loss were classified as aggressive periodontitis (AP) while the remaining subjects over 35 years old who fulfilled our case recruitment criteria and self-reported nil periodontal disease history before 35 years were classified as chronic periodontitis (CP). Controls were Chinese patients aged 18 to 60 years without periodontitis, according to the following criteria: the OPG taken at recruitment showed no sites with more than $15 \%$ bone loss or any radiographic evidence of furcation involvement (each tooth contributed to a mesial and a distal site), subsequent clinical periodontal examination confirmed there were 
no sites with a probing depth of more than $4 \mathrm{~mm}$ and gingival recession of more than $2 \mathrm{~mm}$, and there was no history of tooth loss due to periodontal diseases. Control or periodontitis-free subjects included individuals periodontally health and those with gingivitis. Bleeding on probing (BOP) was not recorded in the control group.

\section{DNA isolation and genotyping}

Ten millilitres of venous blood was obtained from each participant and stored in ethylene-diamine-tetra-acetic acid at $-70^{\circ} \mathrm{C}$ until DNA extraction. QIAamp DNA blood mini-kits (Qiagen, Hilden, Germany) were used to extract genomic DNA. Tagging SNPs of the C5 gene were selected by using the SNP Tagging Wizard of SNPbrowser software version 3.5 (Applied Biosystems, Foster City, CA, USA). The reason to choose tagging SNPs was to select a minimum informative subset of SNPs and eliminate redundant information due to strong linkage disequilibrium among the SNPs in the region. Therefore the SNPs studied here can provide some level of information of the SNPs not selected in this study. Tagging SNPs were those left after eliminating all the SNPs with reported minor allele frequencies under $10 \%$ and reported $100 \%$ linked with other SNPs in the same region. SNP sequences were checked in the RealSNP Assay Database (Sequenom, San Diego, CA, USA). Genotyping was performed with the i-plexGOLD genotyping assay of the 
MassARRAY mass-spectrometry system, following the protocol recommended by the manufacturer (Sequenom).

\section{Quality control}

As recommended in the genotyping protocol, all template DNA samples needed to have a ratio of spectrophotometer readings at $260 \mathrm{~nm}$ and $280 \mathrm{~nm}\left(\mathrm{~A}_{260} / \mathrm{A}_{280}\right)$ of between 1.7 and 2.0. Samples were then aliquoted in 96-well plates. For each well of DNA to be tested, five duplicate wells and one water well served as internal and negative controls, respectively. Before genotyping, DNA samples and randomly selected positive controls were subjected to electrophoresis in $1 \%$ agarose gel to confirm the quality and quantity of genomic DNA. After genotyping by iPLEX, 25 random samples of selected SNPs were subjected to concordance testing by direct sequencing to ensure that the genotyping data were reliable.

\section{Statistical analyses}

Statistical analysis was performed with the Statistical Package for the Social Sciences 15.0 (SPSS, Chicago, IL, USA). Differences between the distributions of sex, and reported smoking habit among periodontitis and periodontitis-free individuals were tested with the chi-square test. Differences between cases and controls in number of 
standing teeth and age were assessed with the $t$ test.

Three screening steps were adopted for genetic data processing. First, the genotype distribution and allele-type of each SNP among AP or CP and periodontitis-free subjects were calculated. Secondly, those SNPs with call rate over $80 \%$ and minor allele frequencies (MAFs) over $10 \%$ were put into the second step: Hardy-Weinberg Equilibrium (HWE) test. Thirdly, those SNPs in the periodontitis-free group with HWE test $p$ values $\geq 0.01$ were subjected to final statistical analysis. Multiple comparison of genotype and allele-type of AP, CP and control groups were initially screened by Chi square test. SNPs fulfilling criteria for final statistical analysis was put under stepwise logistic regression analysis together with other possible risk indicators of periodontitis - namely, age, sex, and reported smoking habit. The alpha level was set at 0.05 , unless otherwise specified. Risk alleles, odds ratios (ORs), and 95\% confidence intervals for each SNP were also determined. Linkage disequilibrium (LD) analysis and haplotype association analysis were performed with Haploview 3.32 (http://www.broad.mit.edu/mpg/haploview/). Haplotype effects in different genetic models were tested by HAPSTAT 3.0 (http://www.bios.unc.edu/ lin/hapstat/).

\section{Results}


A total of 436 participants were recruited: 229 patients with at least moderate periodontitis defined earlier and 207 periodontitis-free controls. The mean age $( \pm$ SD) age of the participants was $43.1 \pm 7.7$ years; $249(57.1 \%)$ subjects were women. There were no differences in mean age and sex distributions between patient and control groups. The case group had about twice the proportion of smokers as did the control group and significantly fewer standing teeth (Table 1). Clinical parameters such as probing pocket depth $(\mathrm{PPD}) \geq 5 \mathrm{~mm}$, probing attachment level $(\mathrm{PAL}) \geq 5 \mathrm{~mm}$, and BOP\% were also summarized in Table 1.

Eleven tagging SNPs of the C5 gene were identified as candidate SNPs by the SNP Tagging Wizard of SNPbrowser 3.5. Most of them were intronic SNPs. Ten of the 11 SNPs were genotyped successfully by the iPLEX assay and seven were found to have more than one genotype (minor allele frequency $>0.001$ ) with an average call rate of $99.2 \%$. One SNP (rs10818491) was found not fulfilling the Hardy-Weinberg Equilibrium $(p<0.01)$ (Table 2$)$, hence analyze of the genotype and allele-type distribution differences of the remaining six SNPs among subgroups like AP, CP and controls was carried out. None of the 6 SNPs showed significant allele or genotype distribution difference between either patient group vs control group (adjusted $p$-value 0.008) (Table 3), which indicated there was no difference between AP, CP and control groups. Therefore, in the following stepwise logistic regression analysis, $\mathrm{AP}$ and $\mathrm{CP}$ 
data were combined.

The six C5 SNPs fulfilling criteria for genetic analysis (Table 3), together with age, sex and smoking habit were selected for stepwise logistic regression analysis regarding the association between these possible risk indicators and periodontitis.

After the adjustment of age, sex and smoking, only rs17611 and smoking habit remained to be significantly associated with periodontitis $(p=0.023$ and 0.006 , respectively, Table 4), genotype AG of rs17611 seemed more frequent in periodontitis $(p=0.007, \mathrm{OR}=6.08, \mathbf{9 5 \%} \mathbf{C I}=\mathbf{1 . 3 1 - 2 8 . 2 2})$. Smoking was also significantly associated with periodontitis in this Hong Kong Chinese sample with OR $=2.84$ $(95 \%$ CI = 1.31-6.14, Table 4).

Haploview 3.32 was used to detect any LD block and the association between haplotypes and periodontitis (19) for the six SNPs that had a Hardy-Weinberg Equilibrium test $p$ value of larger than $10^{-5}$, a call rate of $>0.8$, and a minor allele frequency of $>0.001$. The pairwise comparisons were designed to identify markers within $500 \mathrm{~kb}$ from each other, and one LD block including SNPs rs1035029, rs17611, rs25681 and rs992670 were detected (Figure 1). The haplotype CGCA in this block was found to be marginal significantly associated with periodontitis $(p=0.038$; Table 5). Then HAPSTAT 3.0 was used to estimate haplotype effects and haplotype-environment interactions (age, sex and smoking habit in particular in this 
study) under different genetic models such as dominant and additive models (20).

While dominant genetic model means heterozygote has the same increased risk as minor homozygous genotypes (21), additive model is a statistical model modified from several regression models (22). No environment or haplotype - environmental interaction was found significantly associated with periodontitis. Haplotype CGCA was found significantly associated with periodontitis in the dominant model ( $p=$ 0.001, $\mathrm{OR}=4.85, \mathbf{9 5 \%} \mathbf{C I}=\mathbf{1 . 8 5 - 1 2 . 7 1})($ Table 5). Other genetic models were not suitable for our dataset therefore were not calculated.

\section{Discussion}

It is well-known that complement system is a biochemical cascade helping clear pathogens from an organism. Humoral activation of the complement system by the classical, alternative, or lectin pathway results in the cleavage of $\mathrm{C} 3$ into $\mathrm{C} 3 \mathrm{a}$ and $\mathrm{C} 3 \mathrm{~b}$ fragments. Subsequent downstream cleavage of C5 generates the anaphylatoxin C5a and fragment $\mathrm{C} 5 \mathrm{~b}$, which acts as a nucleus for the MAC (23) by anchoring the assembly of a molecule each of $\mathrm{C} 6, \mathrm{C} 7$, and $\mathrm{C} 8$, to guide the polymerization of $\mathrm{C} 9$ into a membrane channel in the target cell (24). Not only does pore formation cause direct cell injury and necrosis (25), but it may also amplify the inflammatory response by promoting the expression of pro-inflammatory mediators (26). Additionally, the 
MAC can influence the recruitment of inflammatory cells and leukocyte adhesion to endothelium $(27,28)$, thereby promoting the release of cell stimulants, such as hydrolytic enzymes, reactive oxygen species, and cytokines (29, 30). Hence, mutations in any of the MAC complement components might modify this inflammatory response to pathogens. In this study of Hong Kong Chinese patients with and without periodontitis, genotype AG of non-synonymous SNP rs17611 in the C5 gene and the haplotype containing rs17611 were found to be significantly associated with periodontitis, indicating that this genetic variation and haplotype might play a role in the pathogenesis of periodontitis.

The C5 gene is located on chromosome 9q34.1. Eleven SNPs were selected in this study. Although most of the SNPs selected were in intron and their biological function was unclear, but nature of other regional SNPs can be obtained through investigation of their linkage and haplotype status with those tagging SNPs. Therefore information about SNPs searching range and hence the true causative variation(s) could potentially be identified in subsequent experiments. The haplotype and tagging polymorphisms of C5 have been studied in diseases such as liver fibrosis, rheumatoid arthritis, and asthma (11-16). Haplotype included the non-synonymous SNP rs17611 (A/G) in exon 19 was reported to associate with bronchial asthma and liver fibrosis $(12,14)$. The A to $\mathrm{G}$ variation that was found to be 
associated with periodontitis in this study causes an amino acid change from isoleucine to valine at position 802 . Because these two amino acids are both aliphatic and their structures are similar except for an extra center of asymmetry in the isoleucine side chain, the change from isoleucine to valine can be considered to be a conservative one.

Classically, it has been assumed that a mutant protein is unstable and leads to a functional defect, so subjects carrying a homozygous mutant genotype would be more susceptible than others to disease. However, a mutated gene may also encode a stable mutant protein that interferes with the formation of a functional form of the wild-type protein especially for structure proteins or proteins forming dimers (31). An individual carrying a heterozygous genotype in this situation would be more susceptible than one carrying homozygous genotypes $(31,32)$ because products from both genotypes are stable. In this way, the protein with the conservative mutation for valine in position 802, which is expected to be stable, might theoretically affect the wild-type protein. The amino acid mutation in rs17611 is in the MG6 domain of C5, which forms a conserved large cavity with other domains (33). Although the amino acid is far from the cleavage site, this cavity area may be involved in recognition of $\mathrm{C} 5$ by the cleavage enzyme C5 convertase, which is a large macromolecular complex that could conceivably interact with this domain (34). The alteration in rs17611 could also 
potentially affect the formation of the C5b-9 MAC because the substituted amino acid would theoretically be situated within the C5b peptide after cleavage. Further studies on the functions of this gene variant and its effect on periodontitis risk are required. Additionally, the significant association in dominant model of haplotype consisting of SNPs rs1035029, rs17611, rs25681, and rs992670 indicates that SNPs within this gene region could be associated with periodontitis, and further investigation should focus on this particular region.

Smoking is a well-established risk factor in the incidence and progression of periodontal diseases (35-39). One community study conducted among the Hong Kong population showed that heavy smoking is the major risk factor of periodontitis (odds ratio, $\mathrm{OR}=4.61$, and that moderate and light smoking are also significant risk factors $(\mathrm{OR}=2.69$ and 2.33, respectively) (40). These values are similar to the OR (95\% CI) of 2.84 (1.31-6.14) calculated in our study. Nevertheless, we could not subcategorize the small number of smokers as heavy, moderate, light, and very light smokers, which may explain why the OR for smoking is lower than the published value for heavy smoking. The value is also lower than the OR (95\% CI) of 6.08 (1.31-28.22) calculated for the rs17611 genetic variant in this study. It must be noted, however, that our strict criterion for a case was any patient with at least moderate periodontitis, and smoking and the rs17611 genetic variant could have a different influence on mild to 
moderate periodontitis. Finally, other factors such as age and sex have been reported to be associated with periodontitis in Hong Kong Chinese patients (40), but the effects of these two factors were removed by our case-control study design.

It is noticed that in our study we used extra-oral radiographs instead of intraoral radiograph although OPG had only a sensitivity of $79 \%$ for periodontal pathology (41). Nevertheless, OPG has reported a specificity of $92 \%$ for periodontal pathology, and studies have confirmed that bone loss measured on OPG is in close correlated with periodontal status $(42,43)$. In our study, a simple and robust method must be adopted in order to screen a large number of subjects and to eliminate any ambiguous subjects whose periodontal condition did not fulfill recruitment criteria of either groups, therefore OPG was selected for assessing bone loss level of the subjects. We used Schei ruler without using the $1 \mathrm{~mm}$ space or what was referred to be the "normal" distance from alveolar crest to CEJ, instead a certain level or fraction of "bone loss" was allowed for the control group, reason being that OPG typically magnify tooth length ranges from $15-30 \%(44,45)$. Considering average permanent tooth root length is about $11 \mathrm{~mm}$ to $17 \mathrm{~mm}$ (46), $1 \mathrm{~mm}$ equals to about 5-9\% root length from CEJ to radiographic apex. In order to provide some leeway for measurement, we used $15 \%$ root length as the cut off when some other study considered $2 \mathrm{~mm}$ as the "normal" distance between the alveolar crest to CEJ (47). All 
these subjects selected by OPG screening were subsequently periodontally examined to confirm their eligibility as per our inclusion criteria.

In conclusion, our study showed that genotype AG of non-synonymous SNP rs17611 of the $\mathrm{C} 5$ gene was more prevalent in the periodontitis patient group than in periodontitis-free controls and together with smoking may be associated with periodontitis among the Hong Kong Chinese population. In addition, one haplotype including SNP rs17611 was associated with periodontitis. An association study with a large sample size and other ethnic populations are needed to confirm this finding. Further studies should also investigate how genetic variation may alter the structure and biological function of $\mathrm{C} 5$ and its fragments, and how these alterations modify humoral immune responses and susceptibility to periodontitis among the Hong Kong Chinese population, and possibly other populations.

\section{Acknowledgements}

This study was supported by University Research Committee grants 10206094 and 10207348. We thank Trevor Lane for manuscript editing, Alan Wong for technical assistance during sequencing and Bernard Liang for orthopantomographs screening. 


\section{References}

(1) Yoshie H, Kobayashi T, Tai H, Galicia JC. The role of genetic polymorphisms in periodontitis. Periodontol 2000 2007; 43: 102-132.

(2) Carroll MC. The complement system in regulation of adaptive immunity. Nat Immunol 2004; 5: 981-986.

(3) Kohl J, Wills-Karp M. Complement regulates inhalation tolerance at the dendritic cell/T cell interface. Mol Immunol 2007; 44: 44-56.

(4) Schenkein HA, Genco RJ. Gingival fluid and serum in periodontal diseases. II. Evidence for cleavage of complement components $\mathrm{C} 3, \mathrm{C} 3$ proactivator (factor B) and C4 in gingival fluid. J Periodontol 1977; 48: 778-784.

(5) Niekrash CE, Patters MR. Simultaneous assessment of complement components $\mathrm{C} 3, \mathrm{C} 4$, and $\mathrm{B}$ and their cleavage products in human gingival fluid. I. Reliability of the method. J Periodontal Res 1985; 20: 260-267.

(6) Niekrash CE, Patters MR. Assessment of complement cleavage in gingival fluid in humans with and without periodontal disease. J Periodontal Res 1986; 21: $233-242$.

(7) Seppanen M, Lokki ML, Notkola IL, et al. Complement and C4 null alleles in severe chronic adult periodontitis. Scand J Immunol 2007; 65: 176-181.

(8) Gerard NP, Gerard C. The chemotactic receptor for human C5a anaphylatoxin. Nature 1991; 349: 614-617.

(9) Gerard C, Gerard NP. C5a anaphylatoxin and its seven transmembrane-segment receptor. Annu Rev Immunol 1994; 12: 775-808.

(10) Podack ER, Esser AF, Biesecker G, Muller-Eberhard HJ. Membrane attack complex of complement: a structural analysis of its assembly. J Exp Med 1980; 151: 301-313.

(11) Karp CL, Grupe A, Schadt E, et al. Identification of complement factor 5 as a susceptibility locus for experimental allergic asthma. Nat Immunol 2000; 1: 221-226. 
(12) Hasegawa K, Tamari M, Shao C, et al. Variations in the C3, C3a receptor, and C5 genes affect susceptibility to bronchial asthma. Hum Genet 2004; 115: 295-301.

(13) Hillebrandt S, Wasmuth HE, Weiskirchen R, et al. Complement factor 5 is a quantitative trait gene that modifies liver fibrogenesis in mice and humans. Nat Genet 2005; 37: 835-843.

(14) Gressner O, Meier U, Hillebrandt S, et al. Gc-globulin concentrations and C5 haplotype-tagging polymorphisms contribute to variations in serum activity of complement factor C5. Clin Biochem 2007; 40: 771-775.

(15) Kurreeman FA, Padyukov L, Marques RB, et al. A candidate gene approach identifies the TRAF1/C5 region as a risk factor for rheumatoid arthritis. PLoS Med 2007; 4: e278.

(16) Plenge RM, Seielstad M, Padyukov L, et al. TRAF1-C5 as a risk locus for rheumatoid arthritis--a genomewide study. $N$ Engl J Med 2007; 357: 1199-1209.

(17) Bassiouny MA, Grant AA. The accuracy of the Schei ruler: a laboratory investigation. J Periodontol 1975; 46: 748-752.

(18) Armitage GC. Development of a classification system for periodontal diseases and conditions. Ann Periodontol 1999; 4: 1-6.

(19) Teo YY, Fry AE, Clark TG, Tai ES, Seielstad M. On the usage of HWE for identifying genotyping errors. Ann Hum Genet 2007; 71: 701-703; author reply 704 .

(20) Lin DY, Zeng D, Millikan R. Maximum likelihood estimation of haplotype effects and haplotype-environment interactions in association studies. Genet Epidemiol 2005; 29: 299-312.

(21) Demchuk E, Yucesoy B, Johnson VJ, et al. A statistical model for assessing genetic susceptibility as a risk factor in multifactorial diseases: lessons from occupational asthma. Environ Health Perspect 2007; 115: 231-234.

(22) Hastie T, Tibshirani R. Generalized Additive Models: Chapman \& Hall/CRC, 1990.

(23) Schmidt RE, Gessner JE. Fc receptors and their interaction with complement 
in autoimmunity. Immunol Lett 2005; 100: 56-67.

(24) Fondevila C, Shen XD, Tsuchihashi S, et al. The membrane attack complex (C5b-9) in liver cold ischemia and reperfusion injury. Liver Transpl 2008; 14: 1133-1141.

(25) Biancone L, David S, Della Pietra V, Montrucchio G, Cambi V, Camussi G. Alternative pathway activation of complement by cultured human proximal tubular epithelial cells. Kidney Int 1994; 45: 451-460.

(26) Schonermark M, Deppisch R, Riedasch G, Rother K, Hansch GM. Induction of mediator release from human glomerular mesangial cells by the terminal complement components C5b-9. Int Arch Allergy Appl Immunol 1991; 96: 331-337.

(27) Hattori R, Hamilton KK, McEver RP, Sims PJ. Complement proteins C5b-9 induce secretion of high molecular weight multimers of endothelial von Willebrand factor and translocation of granule membrane protein GMP-140 to the cell surface. J Biol Chem 1989; 264: 9053-9060.

(28) Kilgore KS, Shen JP, Miller BF, Ward PA, Warren JS. Enhancement by the complement membrane attack complex of tumor necrosis factor-alpha-induced endothelial cell expression of E-selectin and ICAM-1. J Immunol 1995; 155: 1434-1441.

(29) Seeger W, Suttorp N, Hellwig A, Bhakdi S. Noncytolytic terminal complement complexes may serve as calcium gates to elicit leukotriene B4 generation in human polymorphonuclear leukocytes. J Immunol 1986; 137: 1286-1293.

(30) Hansch GM, Seitz M, Betz M. Effect of the late complement components C5b-9 on human monocytes: release of prostanoids, oxygen radicals and of a factor inducing cell proliferation. Int Arch Allergy Appl Immunol 1987; 82: 317-320.

(31) Sidransky E. Heterozygosity for a Mendelian disorder as a risk factor for complex disease. Clin Genet 2006; 70: 275-282.

(32) Sanders CR, Ismail-Beigi F, McEnery MW. Mutations of peripheral myelin protein 22 result in defective trafficking through mechanisms which may be common to diseases involving tetraspan membrane proteins. Biochemistry 2001; 40: 9453-9459. 
(33) Fredslund F, Laursen NS, Roversi P, et al. Structure of and influence of a tick complement inhibitor on human complement component 5. Nat Immunol 2008; 9: 753-760.

(34) Sandoval A, Ai R, Ostresh JM, Ogata RT. Distal recognition site for classical pathway convertase located in the $\mathrm{C} 345 \mathrm{C} /$ netrin module of complement component C5. J Immunol 2000; 165: 1066-1073.

(35) Grossi SG, Zambon JJ, Ho AW, et al. Assessment of risk for periodontal disease. I. Risk indicators for attachment loss. J Periodontol 1994; 65: 260-267.

(36) Grossi SG, Genco RJ, Machtei EE, et al. Assessment of risk for periodontal disease. II. Risk indicators for alveolar bone loss. J Periodontol 1995; 66: 23-29.

(37) Johnson GK. Position paper: tobacco use and the periodontal patient. Research, Science and Therapy Committee of the American Academy of Periodontology. J Periodontol 1999; 70: 1419-1427.

(38) Johnson GK, Hill M. Cigarette smoking and the periodontal patient. $J$ Periodontol 2004; 75: 196-209.

(39) Ryder MI. The influence of smoking on host responses in periodontal infections. Periodontol 2000 2007; 43: 267-277.

(40) Ng SK, Leung WK. A community study on the relationship between stress, coping, affective dispositions and periodontal attachment loss. Community Dent Oral Epidemiol 2006; 34: 252-266.

(41) Balis S. Error and accuracy rates of panoramic radiography as a screening method for mass surveying of children. J Public Health Dent 1981; 41: 220-234.

(42) Walsh TF, al-Hokail OS, Fosam EB. The relationship of bone loss observed on panoramic radiographs with clinical periodontal screening. J Clin Periodontol 1997; 24: 153-157.

(43) Persson RE, Tzannetou S, Feloutzis AG, Bragger U, Persson GR, Lang NP. Comparison between panoramic and intra-oral radiographs for the assessment of alveolar bone levels in a periodontal maintenance population. $J$ Clin Periodontol 2003; 30: 833-839. 
(44) Thanyakarn C, Hansen K, Rohlin M, Akesson L. Measurements of tooth length in panoramic radiographs. 1. The use of indicators. Dentomaxillofac Radiol 1992; 21: 26-30.

(45) Thanyakarn C, Hansen K, Rohlin M. Measurements of tooth length in panoramic radiographs. 2: Observer performance. Dentomaxillofac Radiol 1992; 21: 31-35.

(46) Ash M, Wheeler R, Nelson S. Wheeler's dental anatomy, physiology and occlusion, 8th edn. Philadelphia, Pa.: London: Sauders, 2003.

(47) Lanning SK, Best AM, Temple HJ, Richards PS, Carey A, McCauley LK. Accuracy and consistency of radiographic interpretation among clinical instructors in conjunction with a training program. J Dent Educ 2006; 70: 545-557. 


\section{Legend}

Fig. 1. Pairwise linkage disequilibrium (LD) in the C5 gene. All SNPs of C5 assessed in this study. The horizontal bar represents the relative location of each SNP along the chromosome 9, 9q33-q34, and the numbers above the bar are corresponding chromosome positions; SNPs highlighted in green are coding SNPs. Diamonds in the haplotype blocks represent pairwise linkage disequilibrium between all SNPs assessed; the darker the diamond is, the stronger the linkage disequilibrium is between the two SNPs. 
Table 1. Demography and clinical profile of study participants

\begin{tabular}{|c|c|c|c|c|c|c|}
\hline & & Periodontitis-free & Periodontitis & & & \\
\hline Characteristic & Categories & $(n=207)$ & $(n=229)$ & Test & Statistics & $p$-value \\
\hline$\overline{\text { Age (years) }}$ & Mean \pm SD & $42.4 \pm 8.1$ & $43.7 \pm 7.4$ & $\mathrm{t}$ & -1.69 & NS \\
\hline \multirow[t]{2}{*}{ Gender } & Male & $87(42.0)$ & $100(43.7)$ & $\chi^{2}$ & 0.27 & NS \\
\hline & Female & $120(58.0)$ & $129(56.3)$ & & & \\
\hline \multirow[t]{3}{*}{ Smoking habit } & Non-smoker & $191(92.3)$ & $193(84.3)$ & $\chi^{2}$ & 7.15 & $<0.005$ \\
\hline & Smoker & $16(7.7)$ & $36(15.7)$ & & & \\
\hline & Pack-year $($ mean $\pm S D)$ & $4.7 \pm 6.7$ & $16.0 \pm 10.2$ & $\mathrm{t}$ & -3.80 & $<0.05$ \\
\hline Teeth remain & Mean \pm SD & $27.0 \pm 1.8$ & $24.7 \pm 3.4$ & $\mathrm{t}$ & 5.46 & $<0.001$ \\
\hline$\%$ sites $\mathrm{PPD} \geq 5 \mathrm{~mm}$ & Mean \pm SD & $0 \pm 0$ & $30.3 \pm 18.1$ & & & \\
\hline$\%$ sites $\mathrm{PAL} \geq 5 \mathrm{~mm}$ & Mean \pm SD & $0 \pm 0$ & $47.2 \pm 27.2$ & & & \\
\hline BOP \% & Mean $\pm \mathrm{SD}$ & ND & $72.6 \pm 25.0$ & & & \\
\hline
\end{tabular}

Results are no. (\%) unless otherwise indicated. ND: not determined. 
Table 2. Candidate C5 single nucleotide polymorphisms (SNPs) of study participants

\begin{tabular}{llllll}
\hline SNP ID & Type & Variation & Call rate (\%) & MAF & HWE $p$ - \\
& & & & & value $^{\mathrm{b}}$ \\
\hline rs2300930 & Intronic & $\mathrm{A} / \mathrm{C}$ & 98.9 & 0.197 & 0.73 \\
rs10818491 & Intronic & $\mathrm{C} / \mathrm{T}$ & 98.7 & 0.406 & $<10^{-5}$ \\
rs7035682 & Intronic & $\mathrm{C} / \mathrm{T}$ & 99.3 & single genotype detected & \\
rs7026551 & Intronic & $\mathrm{A} / \mathrm{C}$ & 98.4 & single genotype detected & \\
rs2269066 & Intronic & $\mathrm{A} / \mathrm{G}$ & 98.2 & 0.237 & 0.20 \\
rs2269067 & Intronic & $\mathrm{C} / \mathrm{G}$ & 99.1 & single genotype detected & \\
rs1035029 & Intronic & $\mathrm{C} / \mathrm{T}$ & 99.1 & 0.409 & 0.93 \\
rs17611 & Non-synonymous & $\mathrm{A} / \mathrm{G}$ & 99.8 & 0.409 & 0.73 \\
rs25681 & Synonymous & $\mathrm{C} / \mathrm{T}$ & 99.8 & 0.409 & 0.96 \\
rs992670 & Intronic & $\mathrm{A} / \mathrm{G}$ & 99.1 & 0.230 & 0.24 \\
rs2300934 & Intronic & $\mathrm{A} / \mathrm{C}$ & 0 & $\mathrm{ND}$ & \\
\hline
\end{tabular}

HWE: Hardy-Weinberg Equilibrium; MAF: Minor Allele Frequency; ND: not determined ${ }^{a}$ MAF not determined when call rate $<80.0 \%$ or only one genotype detected.

${ }^{\mathrm{b}}$ For periodontitis-free group; HWE $p$-value not determined if call rate $<80.0 \%$ or only one genotype detected; only SNPs with HWE $p$-value $\geq 0.01$ are subjected to further analysis. 
Table 3. Genotype and allele-type of C5 SNPs selected for stepwise logistic regression analysis

\begin{tabular}{|c|c|c|c|c|c|}
\hline \multirow[b]{2}{*}{ SNP ID } & Genotype/ & Count $^{\mathrm{a}}(\%)$ & & & \multirow[b]{2}{*}{$p$-value ${ }^{\mathrm{b}}$} \\
\hline & $\begin{array}{l}\text { Allele- } \\
\text { type }\end{array}$ & $\begin{array}{l}\text { Periodontitis-free } \\
(\mathrm{n}=207)\end{array}$ & $\begin{array}{l}\text { AP } \\
(n=32)\end{array}$ & $\begin{array}{l}\text { CP } \\
(n=197) \\
\end{array}$ & \\
\hline \multirow{5}{*}{ rs2300930 } & $\mathrm{AA}$ & $7(3.4)$ & $1(3.1)$ & $7(3.6)$ & \multirow{3}{*}{0.278} \\
\hline & $\mathrm{AC}$ & 57 (27.9) & $8(25.0)$ & $73(37.4)$ & \\
\hline & $\mathrm{CC}$ & $140(68.6)$ & $23(71.9)$ & $115(59.0)$ & \\
\hline & $\mathrm{A}$ & $71(17.4)$ & $10(15.6)$ & $87(22.3)$ & \multirow{2}{*}{0.156} \\
\hline & $\mathrm{C}$ & $337(82.6)$ & $54(84.4)$ & $303(79.7)$ & \\
\hline \multirow{5}{*}{ rs2269066 } & $\mathrm{AA}$ & $118(57.6)$ & $19(59.4)$ & $110(56.4)$ & \multirow{3}{*}{0.487} \\
\hline & $\mathrm{AG}$ & 75 (36.6) & $11(34.4)$ & $80(41.0)$ & \\
\hline & GG & $12(5.9)$ & $2(6.2)$ & $5(2.6)$ & \\
\hline & $\mathrm{A}$ & $311(75.9)$ & $49(76.6)$ & $300(76.9)$ & 0.938 \\
\hline & $\mathrm{G}$ & $99(24.1)$ & $15(23.4)$ & $90(23.1)$ & \\
\hline \multirow{5}{*}{ rs1035029 } & $\mathrm{CC}$ & $36(17.6)$ & $5(15.6)$ & $30(15.4)$ & \multirow{3}{*}{0.055} \\
\hline & $\mathrm{CT}$ & 87 (42.6) & $13(40.6)$ & $110(56.4)$ & \\
\hline & TT & $81(39.7)$ & $14(43.8)$ & $55(28.2)$ & \\
\hline & $\mathrm{C}$ & $159(39.0)$ & $23(35.9)$ & $170(43.6)$ & 0.294 \\
\hline & $\mathrm{T}$ & $249(61.0)$ & $41(64.1)$ & $220(56.4)$ & \\
\hline \multirow{5}{*}{ rs17611 } & $\mathrm{AA}$ & $82(40.2)$ & $13(40.6)$ & $56(28.7)$ & \multirow{3}{*}{0.053} \\
\hline & $\mathrm{AG}$ & $85(41.7)$ & $14(43.8)$ & $110(56.4)$ & \\
\hline & GG & $37(18.1)$ & $5(15.6)$ & $29(14.9)$ & \\
\hline & $\mathrm{A}$ & $249(61.0)$ & $40(62.5)$ & $222(56.9)$ & 0.430 \\
\hline & $\mathrm{G}$ & $159(39.0)$ & $24(37.5)$ & $168(43.1)$ & \\
\hline \multirow{3}{*}{ rs 25681} & $\mathrm{CC}$ & $37(18.1)$ & $6(18.8)$ & $28(14.4)$ & \multirow{3}{*}{0.033} \\
\hline & CT & $86(42.2)$ & $12(37.5)$ & $111(56.9)$ & \\
\hline & TT & $81(39.7)$ & $14(43.8)$ & $56(28.7)$ & \\
\hline
\end{tabular}




\begin{tabular}{|c|c|c|c|c|c|}
\hline & $\mathrm{C}$ & $160(39.2)$ & $24(37.5)$ & $167(42.8)$ & 0.685 \\
\hline & $\mathrm{T}$ & $248(61.8)$ & $40(62.5)$ & $223(57.2)$ & \\
\hline \multirow{4}{*}{ rs992670 } & AA & $117(57.1)$ & $19(59.4)$ & $115(59.0)$ & \multirow{2}{*}{0.178} \\
\hline & $\mathrm{AG}$ & 75 (36.6) & $11(34.4)$ & $77(39.5)$ & \\
\hline & A & 309 (75.4) & 49 (76.6) & 307 (78.7) & \multirow[t]{2}{*}{0.529} \\
\hline & $\mathrm{G}$ & $101(24.6)$ & $15(23.4)$ & $83(21.3)$ & \\
\hline
\end{tabular}

AP: Aggressive periodontitis; CP: Chronic periodontitis

${ }^{\mathrm{a}}$ Total not adding up because call rates are $<100 \%$.

${ }^{\mathrm{b}}$ The level of significance was set at 0.008 after adjustment for multiple comparison 
Table 4. Odds ratios for study participants with periodontitis

\begin{tabular}{|c|c|c|c|c|c|c|c|c|c|}
\hline \multirow{3}{*}{$\begin{array}{l}\text { Variable } \\
\text { Age }\end{array}$} & \multirow{3}{*}{ Categories } & \multicolumn{4}{|c|}{ Unadjusted (univariate) $^{\mathrm{a}}$} & \multicolumn{4}{|c|}{ Adjusted $^{b}$} \\
\hline & & \multirow{2}{*}{$\begin{array}{r}\text { OR } \\
1.02\end{array}$} & \multirow{2}{*}{$\frac{p \text {-value }}{0.072}$} & \multicolumn{2}{|c|}{$95 \% \mathrm{CI}$} & \multirow[t]{2}{*}{ OR } & \multirow[t]{2}{*}{$p$-value } & \multicolumn{2}{|c|}{$95 \% \mathrm{CI}$} \\
\hline & & & & 0.99 & -1.05 & & & & \\
\hline \multirow[t]{2}{*}{ Gender } & Female & 1.00 & 0.601 & & & & & & \\
\hline & Male & 0.90 & & 0.62 & -1.32 & & & & \\
\hline \multirow[t]{2}{*}{ Smoking habit } & Non-smoker & 1.00 & 0.003 & & & 1.00 & 0.006 & & \\
\hline & Smoker & 2.57 & & 1.34 & -4.92 & 2.84 & & 1.31 & -6.14 \\
\hline \multicolumn{10}{|l|}{ Genotype (SNP) } \\
\hline \multirow[t]{3}{*}{ rs2300930 } & $\mathrm{AA}$ & 1.00 & 0.220 & & & & & & \\
\hline & $\mathrm{AC}$ & 1.24 & 0.690 & 0.43 & -3.62 & & & & \\
\hline & $\mathrm{CC}$ & 0.86 & 0.781 & 0.30 & -2.44 & & & & \\
\hline \multirow[t]{3}{*}{ rs2269066 } & AA & 1.00 & 0.338 & & & & & & \\
\hline & $\mathrm{AG}$ & 1.11 & 0.605 & 0.75 & -1.65 & & & & \\
\hline & GG & 0.53 & 0.202 & 0.20 & -1.40 & & & & \\
\hline \multirow[t]{3}{*}{ rs1035029 } & TT & 1.00 & 0.051 & & & & NS & & \\
\hline & $\mathrm{CT}$ & 1.66 & 0.024 & 1.09 & -2.53 & & & & \\
\hline & $\mathrm{CC}$ & 1.14 & 0.667 & 0.68 & -2.01 & & & & \\
\hline \multirow[t]{3}{*}{ rs17611 } & AA & 1.00 & 0.026 & & & 1.00 & 0.023 & & \\
\hline & $\mathrm{AG}$ & 1.73 & 0.014 & 1.14 & -2.65 & 6.08 & 0.007 & 1.31 & -28.22 \\
\hline & GG & 1.09 & 0.775 & 0.62 & - 1.92 & 1.15 & 0.707 & 0.63 & - 1.97 \\
\hline rs25681 & TT & 1.00 & 0.044 & & & & NS & & \\
\hline
\end{tabular}


rs992670
AA $1.00 \quad 0.120$
$\begin{array}{llllll}\text { AG } & 1.02 & 0.905 & 0.69 & - & 1.52\end{array}$

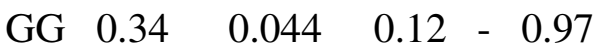

Allele-type

rs2300930

C $\quad 1.00$

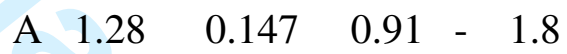

rs2269066

G 1.00

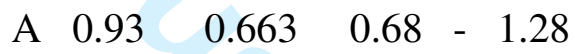

rs1035029

C 1.00

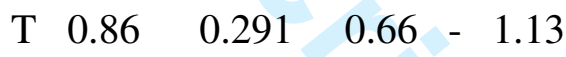

rs 17611

A 1.00

G $\quad 1.15 \quad 0.307 \quad 0.88 \quad-\quad 1.51$

rs 25681

C 1.00

$\begin{array}{llllll}\mathrm{T} & 0.92 & 0.531 & 0.70 & - & 1.20\end{array}$

rs992670

A 1.00

$\begin{array}{lllll}\mathrm{G} & 0.84 & 0.288 & 0.61 & -1.16\end{array}$

$\mathrm{OR}=$ odds ratio $\mathrm{CI}=$ confident interval; $\mathrm{NS}=$ not significant.

${ }^{\mathrm{a}} \mathrm{OR}$ for each factor was computed from logistic regression

${ }^{\mathrm{b}}$ Adjusted ORs were computed with stepwise logistic regression with variables that were significant in the univariate analysis 
Table 5. Haplotype association results in different genetic models $(\mathrm{n}=872)$

\begin{tabular}{|c|c|c|c|c|c|}
\hline Haplotype $^{a}$ & \multicolumn{2}{|c|}{ Count Ratio $^{\mathrm{b}}$ (Frequencies) } & $\chi^{2} p$-value & \multicolumn{2}{|c|}{ Haplotype effects and haplotype-environment interactions ${ }^{\mathrm{c}, \mathrm{d}}$} \\
\hline CGCG & $95.7 / 314.3(0.233)$ & $93.6 / 362.4(0.205)$ & 0.319 & 0.308 & 0.323 \\
\hline TACA & $4.8 / 405.2(0.012)$ & $5.1 / 450.9(0.011)$ & 0.950 & 0.216 & 0.845 \\
\hline
\end{tabular}

${ }^{\mathrm{a}}$ Two haplotypes are not listed due to low frequencies (fractional likelihood frequency $<0.01$ )

${ }^{\mathrm{b}}$ Counts were obtained by summing the fractional likelihoods of each individual for each haplotype.

${ }^{\mathrm{c}}$ The level of significance was set at 0.0125 after adjustment for multiple comparison

${ }^{\mathrm{d}}$ Logistic regression analysis, shown are $p$-values

${ }^{\mathrm{e}}$ Odds Ratio and 95\% confident interval: 4.85 (1.85-12.71) 
Figure 1 $210 \times 297 \mathrm{~mm}(600 \times 600 \mathrm{DPI})$ 